\title{
Vibration Simulation of the cylindrical reservoir shell containing fluid vortex with the help of Vib-Shape software
}

\author{
Hossein Moradi ${ }^{1}$, Farzan Barati2,*
}

${ }^{1}$ Department of mechanics, Hamadan branch, Islamic azad university, Hamadan, Iran

${ }^{2}$ Department of mechanics, Faculty of engineering Hamadan branch, Islamic azad university,

Hamadan, Iran

\begin{abstract}
This paper addresses the simulation of the vibrational behavior of a flat-bottomed cylindrical reservoir in different levels of fluid vortex with the help of Vib-Shape software. The reservoir is equipped with a mixer, which creates fluid vortex and required loading. The compliance of the shell vibration mode shape of the mentioned model obtained from the collected data and simulation in the aforementioned software is compared with the mode shapes derived from the Donnell's theory. The results are in the good agreement with the governing theories of the problem.
\end{abstract}

Keywords: Vibration, cylindrical reservoir, mixer, vortex fluid, mode shape. 


\section{Introduction}

$\mathrm{T}$ he dynamic behavior of the cylindrical reservoirs filled with fluid has a lot of applications in the industries where the cylindrical reservoirs are used for maintenance or storage of the liquids. When the vortex and rotation of the fluid is considered with the variable level of the fluid simultaneously, the effect of inertial coupling between the fluid and wall motion of the reservoir on the dynamic behavior and stability of the reservoir become more prominent. Although numerous papers have been published about cylindrical reservoirs empty or fluid-filled, or submerged in the fluid, this type of simulation about cylindrical reservoirs with variable fluid level, which contains vortexes due to the mixer, have not been carried out as required. The aim of this research is to simulate the vibration mode shape of the cylindrical reservoir resulting from the existence of vortex fluid. In addition, the compliance with theoretical results of Donnell's theory is checked.

\section{The governing theory - Donnell's theory of shells}

The equation of the motion in the linear vibration of the circular cylindrical shell can be obtained by eliminating the nonlinear terms in the nonlinear equation of the motion.

The equations of the motion in the Donnell's theory are as follows:

$$
R^{2} \frac{\partial^{2} u}{\partial x^{2}}+\frac{1-v}{2} \frac{\partial^{2} u}{\partial \theta^{2}}+R \frac{1+v}{2} \frac{\partial^{2} v}{\partial x \partial \theta}-v R \frac{\partial w}{\partial x}=\rho \frac{1-v^{2}}{E} R^{2} \frac{\partial^{2} u}{\partial t^{2}}
$$

$$
\frac{\partial^{2} v}{\partial \theta^{2}}+R^{2} \frac{1-v}{2} \frac{\partial^{2} v}{\partial x^{2}}+R \frac{1+v}{2} \frac{\partial^{2} u}{\partial x \partial \theta}-\frac{\partial w}{\partial \theta}=\rho \frac{1-v^{2}}{E} R^{2} \frac{\partial^{2} v}{\partial t^{2}}
$$

$$
\begin{aligned}
& v R \frac{\partial u}{\partial x}+\frac{\partial v}{\partial \theta}-w-k\left(R^{4} \frac{\partial^{4} w}{\partial x^{4}}+2 R^{2} \frac{\partial^{4} w}{\partial x^{2} \partial \theta^{2}}+\frac{\partial^{4} w}{\partial \theta^{4}}\right) \\
& =\rho \frac{1-v^{2}}{E} R^{2} \frac{\partial^{2} w}{\partial t^{2}}
\end{aligned}
$$

Where:

$$
k=(1 / 12)(h / R)^{2}
$$

$\mathrm{X}, \mathrm{r}$ and -represents a cylindrical coordinate system, $R$ indicates the radius of the shell, $v$ is the Poisson's ratio and $\mathrm{E}$ is the Young's modulus.

The answer is in the following form:

$$
\begin{aligned}
& u=C_{1} \cos \left(\lambda_{m} x\right) \cos (n \theta) \cos (\omega t) \\
& v=C_{2} \sin \left(\lambda_{m} x\right) \sin (n \theta) \cos (\omega t)
\end{aligned}
$$

$$
w=C_{3} \sin \left(\lambda_{m} x\right) \cos (n \theta) \cos (\omega t)
$$

Where the $\lambda_{\mathrm{m}}=\mathrm{m} \pi / \mathrm{L}, \quad \mathrm{m}=1,2, \ldots \quad$ and $\mathrm{n}=0,1,2, \ldots$. According to the figures 1 and $2, \mathrm{~m}$ and $\mathrm{n}$ are the numbers of axial and circumferential half-waves, respectively. Also, $\omega$ is the free angular frequency and $t$ is the time.
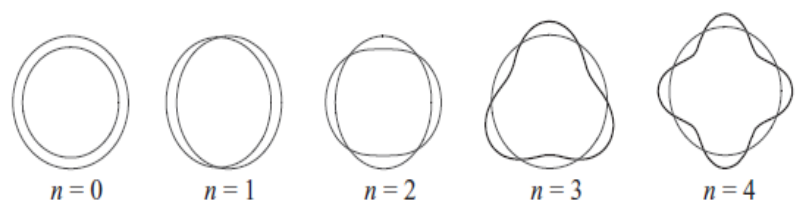

Figure1. The number of circumferential waves $(n)$ determining the mode shapes.
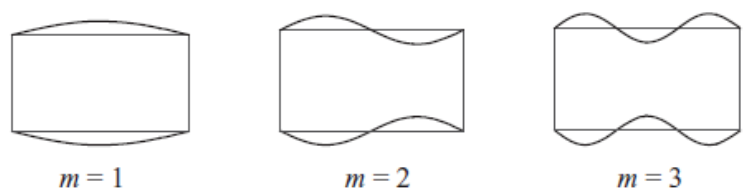

Figure2. The number of axial half-waves (m) determining the mode shapes. 
Equations (2 a,b,c) describe the modes shape $C_{i}, i=1,2,3$ are the constant coefficients related to the domain. For every asymmetric mode shape (ie, for $\mathrm{n}>0$ ) a secondary mode exists, which resembles the primary mode, but is perpendicular to it. As a result, the sine and cosine functions in the angular coordinate $(\theta)$ are transformed by $\pi / 2 n$ (according to the figure 3). This secondary mode causes the maximum vibrational domain accrues in the place, where the first mode has one node.

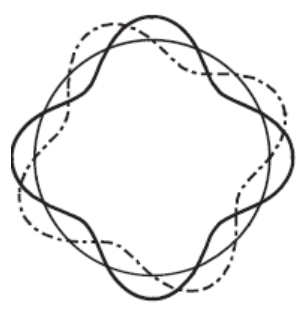

Figure 3. Identical asymmetric modes couple; integrated solid line: cosine mode; dashed line: sine mode; Trimmed line: nondeformed shell

\section{Description of the reservoir}

A thin-walled cylindrical shell (closed bottom and open end) with the radius of 105.38 $\mathrm{mm}$, length of $345 \mathrm{~mm}$, wall thickness of 3.7 $\mathrm{mm}$ and the fluid density of $\rho \mathrm{f}=1000 \mathrm{~kg} / \mathrm{m} 3$, is considered. The cylindrical shell is made from S.S with the mechanical properties of $\mathrm{osh}=700$ $\mathrm{kg} / \mathrm{m} 3, v=0.3$ and $\mathrm{E}=200 \mathrm{Gpa}$.
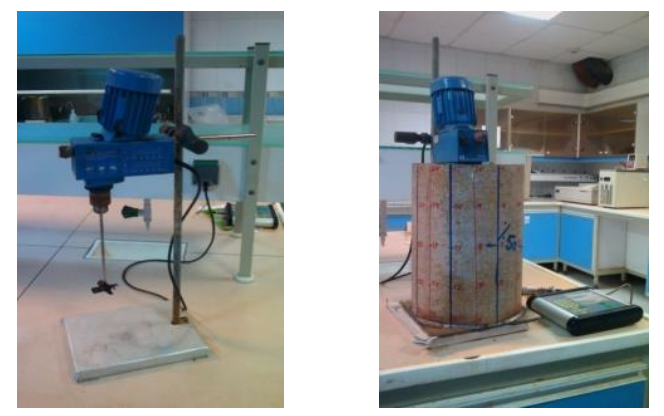

Figure 4. The cylindrical reservoir with a mixer

The mixer propeller (below figure) is located in the center of the cylindrical shell and due to the structure, the Pitched Blade Turbine have pumping action in the axial/radial direction. So, the resulting Flow Rangeis in the turbulent/transition range.
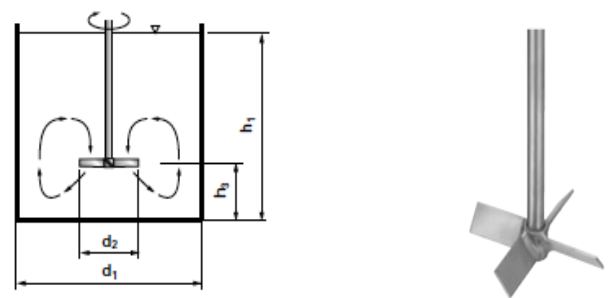

Figure 5. The mixing propeller and it's location

The reservoir pressure is atmospheric pressure. The fluid in the reservoir is nonviscous and incompressible. In addition, the vibration of the plate of the reservoir bottom due to the mixer support is neglected. Also, the distortion of the plate and the shell, and their effect on the vibrational behavior of the reservoir is ignored.

\section{Experiment schedule}

The vibration test of the mentioned reservoir in the cited condition is performed in three following cases:

1- The reservoir filled with the fluid up to $30 \%$ of the height and the rotational speed of 2000 RPM.

2- The reservoir filled with the fluid up to $50 \%$ of the height and the rotational speed of 2000 RPM.

3- The reservoir filled with the fluid up to $75 \%$ of the height and the rotational speed of 2000 RPM.

\section{Location of the sensor}

80 points are determined for measuring the radial motion of the cylindrical shell under the radial loading of the fluid vortex and the resulting flow, which have been shown on the reservoir.

During the test, the amount of the vibration and radial motion of these points is measuredbyan acceleration transducer in each of the three determined cases. 


\section{Data processing and modal identification}

The data for the vibrational behavior of the cylindrical shell is captured with Easy-Viber Vibration Data Collector and then examined in the Vib-Shape and Spectra-Pro environment.

The simulated model of the reservoir is as the figure 6. In the right figure, Corner Point Numbers, and in the left one, the Corner Point Directions are presented. 80 points were specified for measuring the radial motion of the cylindrical shell under the radial loading of the fluid vortex and the resulting flow. The measuring unit for the radial motion is $\mu \mathrm{m}$.

The image of the Vib-Shape environment, which shows the animation of the shell in a moment, is presented for each of the three experiments.

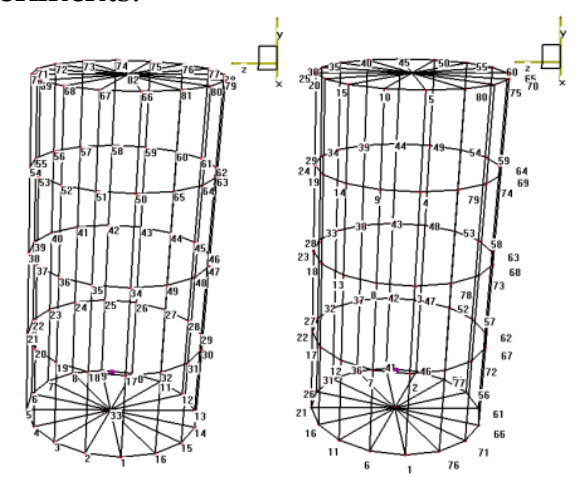

Figure 6. The simulated model of the reservoir. right figure, Corner Point Numbers, and in the left one, the Corner Point Directions.

The image of the Vib-Shape environment, which shows the animation of the shell in a moment for Deviation Anim. Max condition are presented for each case in the figures 7,8 and 9.

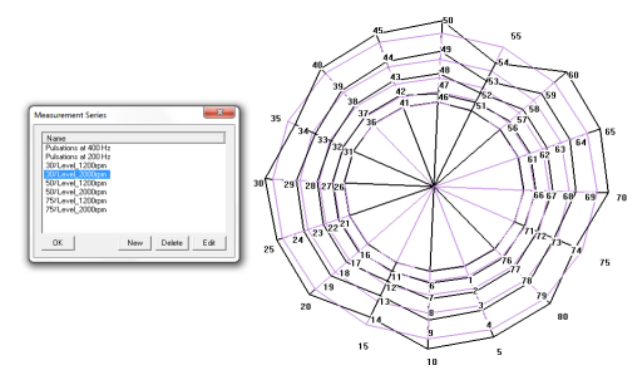

Figure 7. Case 1-The reservoir filled with the fluid up to $30 \%$ of the height and the rotational speed of $2000 \mathrm{rpm}$.

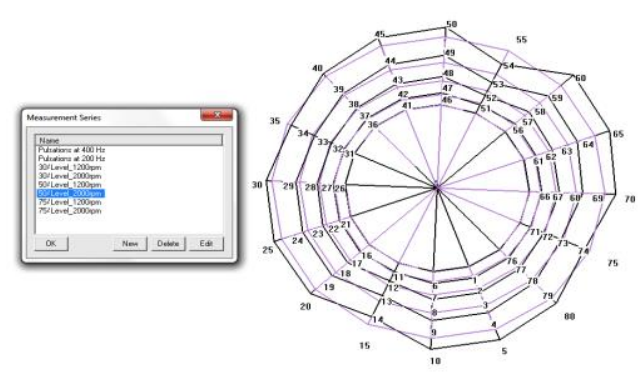

Figure 8. Case 2- The reservoir filled with the fluid up to $50 \%$ of the height and the rotational speed of $2000 \mathrm{rpm}$.

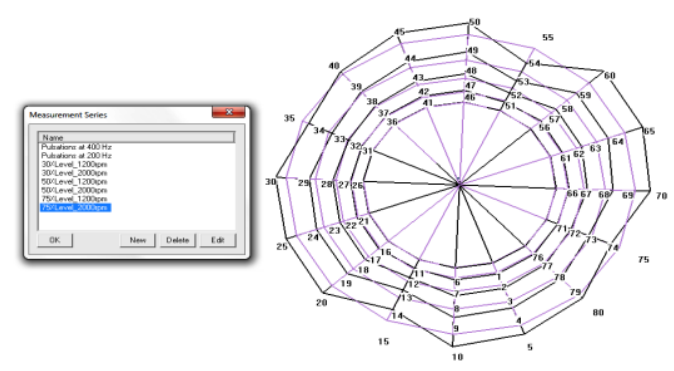

Figure 9. Case 3- The reservoir filled with the fluid up to $75 \%$ of the height and the rotational speed of $2000 \mathrm{rpm}$.

\section{The obtained results due to the vibrational mode shape of the cylindrical reservoir}

The following results are obtained in the three case of the experiments:

1- In the case 1 , the obtained mode shape has sufficient symmetry and relatively good compliance with the results of Donnell's theory (according to figures 3 and 5). The vibration mode represents $\mathrm{n}=4$ and sine mode $($ mode $=\operatorname{Sin} n \theta)$.
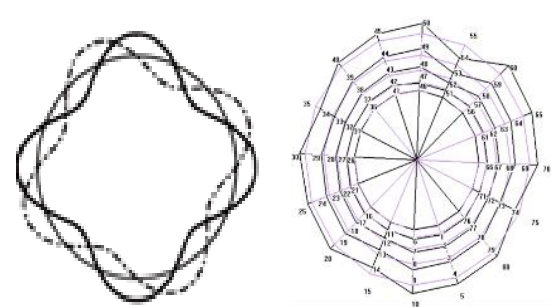

2- In the case 2, the obtained mode shape has relatively good symmetry and fairly close compliance with the results 
of Donnell's theory (according to figures 3 and 6). The vibration mode represents $\mathrm{n}=4$ and sine mode $($ mode $=\operatorname{Sin} n \theta)$.
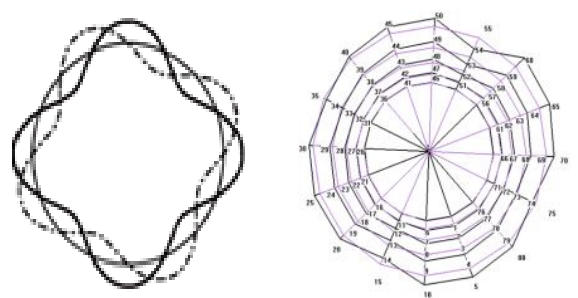

3- In the case 3, the obtained mode shape has sufficient symmetry and relatively good compliance with the results of Donnell's theory (according to figures 3 and 7). The vibration mode represents $\mathrm{n}=4$ and sine mode ( mode $=\operatorname{Sin} n \theta$ ).
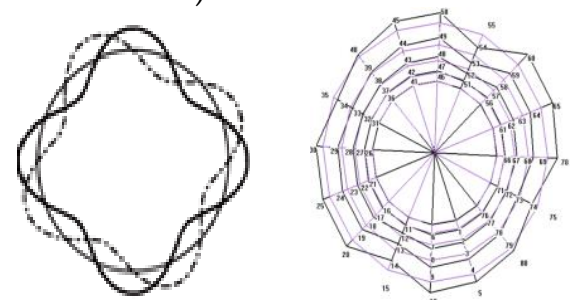

4- Increasing the fluid level in the reservoir in the constant mixing rotation, the vibration value is increased in some points compared to the lower fluid level (Except some points).

So, it can be concluded that the obtained results have adequate accuracy and are consistent with the Donnell's theory of shells.

\section{Suggestions}

Due to extensive use of storage and pressurized reservoirs in various industries, particularly oil, gas and petrochemical industry, and also according to the key role of these equipments in terms of production, process and safety issues in the cases dealing with the high process temperatures and pressures, it is suggested that the vibration behavior of these equipments would be studied in the case of more weld seams, also investigating the vibration mode shapes in this case is suggested.
Amabili ,Marco, (2008), “Nonlinear vibrations and stability of shell and plates", New York, Cambridge University Press.

Askhari E., and Daneshmand F. ,(2009), “Coupled Vibration of a Partially Fluid-Filled Cylindrical Container with a Cylindrical Internal Body", Journal of Fluids and Structures, Vol. 25, 389-405.

Gonçalves Paulo B., N. del Prado Zenón J. G. ,(2010)," Nonlinear Vibrations of Axially Loaded Cylindrical Shells Partially Filled With Fluid",Journal of Brazilian Conference on Dynamic, 9 th,635-643.

Leissa, Arthur W.,(1973),"'Vibration of Shell",Washington D.C,National Aeronautics and Space administration.

Michael P. Païdoussis , (2014)," Fluid-Structure InteractionsSlender Structuresand Axial Flow", Second edition, Volume 1, Elsevier Academic Press.

Michael P. Païdoussis, (2004)," Fluid-Structure InteractionsSlender Structuresand Axial Flow", Second edition, Volume 2, Elsevier Academic Press.

Strutt H. and Runzi D., (2000),"Handbook of EKATO mixing technology", Schopfheim, Germany,EKATO Ruhr-Und Mischtechnik $\mathrm{GmbH}$

\section{References}

